\title{
Spatial Circular Granulation Method Based on Multimodal Finger Feature
}

\author{
Jinfeng Yang, Zhen Zhong, Guimin Jia, and Yanan Li \\ Tianjin Key Lab for Advanced Signal Processing, Civil Aviation University of China, CAUC, Tianjin 300300, China \\ Correspondence should be addressed to Jinfeng Yang; jfyang@cauc.edu.cn
}

Received 2 December 2015; Revised 3 February 2016; Accepted 14 March 2016

Academic Editor: Sook Yoon

Copyright ( 2016 Jinfeng Yang et al. This is an open access article distributed under the Creative Commons Attribution License, which permits unrestricted use, distribution, and reproduction in any medium, provided the original work is properly cited.

\begin{abstract}
Finger-based personal identification has become an active research topic in recent years because of its high user acceptance and convenience. How to reliably and effectively fuse the multimodal finger features together, however, has still been a challenging problem in practice. In this paper, viewing the finger trait as the combination of a fingerprint, finger vein, and finger-knuckle-print, a new multimodal finger feature recognition scheme is proposed based on granular computing. First, the ridge texture features of FP, FV, and FKP are extracted using Gabor Ordinal Measures (GOM). Second, combining the three-modal GOM feature maps in a color-based manner, we then constitute the original feature object set of a finger. To represent finger features effectively, they are granulated at three levels of feature granules (FGs) in a bottom-up manner based on spatial circular granulation. In order to test the performance of the multilevel FGs, a top-down matching method is proposed. Experimental results show that the proposed method achieves higher accuracy recognition rate in finger feature recognition.
\end{abstract}

\section{Introduction}

Biometrics based personal authentication is drawing increasing attention in both academic research and industrial applications. Among current biometric identifiers, finger-based biometrics is widely used because of its high user acceptance and convenience. Some commonly used finger-based biometrics, such as fingerprint $[1,2]$, finger vein [3-5], and fingerknuckle-print $[6,7]$, have been widely used in personal identification. However, many unimodal biometric-based systems have demonstrated that using a single finger-based biometric source for personal identification usually was far from perfect in many real applications. Compared with unimodal biometric approaches, multimodal biometrics always behave better in universality, accuracy, and security [8-10]. How to reliably and effectively fuse the multimodal features together, however, has still been a challenging problem in practice. In recent years, the research on granular computing $(\mathrm{GrC})$ has attracted many researchers and practitioners. The basic idea of $\mathrm{GrC}$ is using information granules during complex problem solving, and granulation is one of key issues in $\mathrm{GrC}$ $[11,12]$. Since Zadeh and Lin first proposed the concept of
GrC [11], many related applications have been proposed [1316]. Firstly, Zheng et al. proposed a tolerance granular space model (TGSM) to study the problem of image segmentation $[13,14]$. Then, Li and Meng proposed a method for MRI and MRA image fusion based on tolerance granular space modal [15]. Further, Bhatt et al. proposed face granulation scheme at multiple levels of granularity for face recognition [16]. These works imply that $\mathrm{GrC}$ is a new way to deal with the complex multimodal biometrics recognition problems.

In this paper, viewing fingerprint (FP), finger vein (FV), and finger-knuckle-print (FKP) as the constitutions of finger trait, we adopt a novel method to study the problems of multimodal finger feature recognition according to the spatial circular granulation. As we know, ridge texture information dominates over these three biometrics characteristics with a compatible feature space. Therefore, a finger itself can be viewed as a coarse granularity information granule with plenty of ridge texture, and each finger feature granule contains different discriminating information forming the finger image signature.

In the proposed method, we adopt the Gabor Ordinal Measures (GOM) $[17,18]$ for multimodal finger feature 

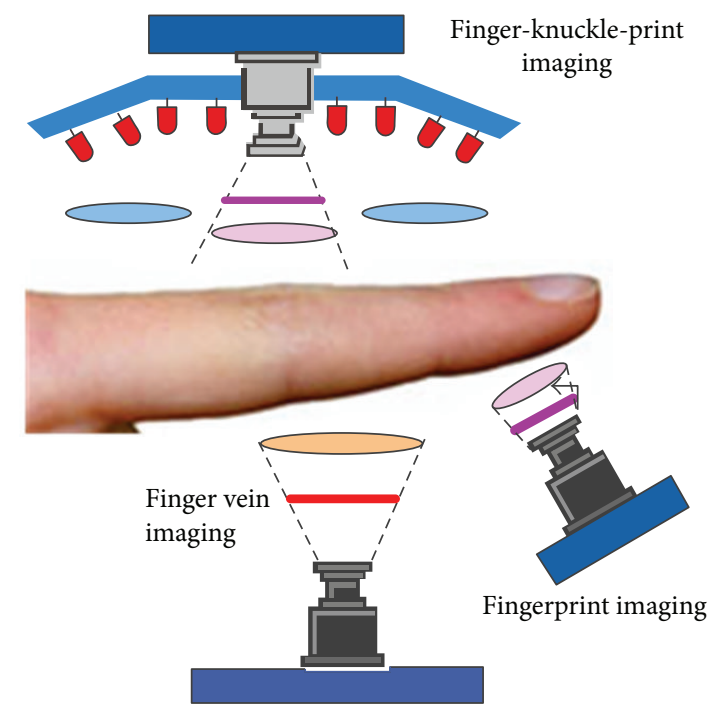

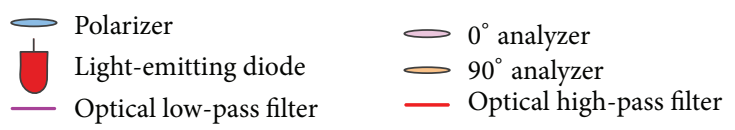

(a)

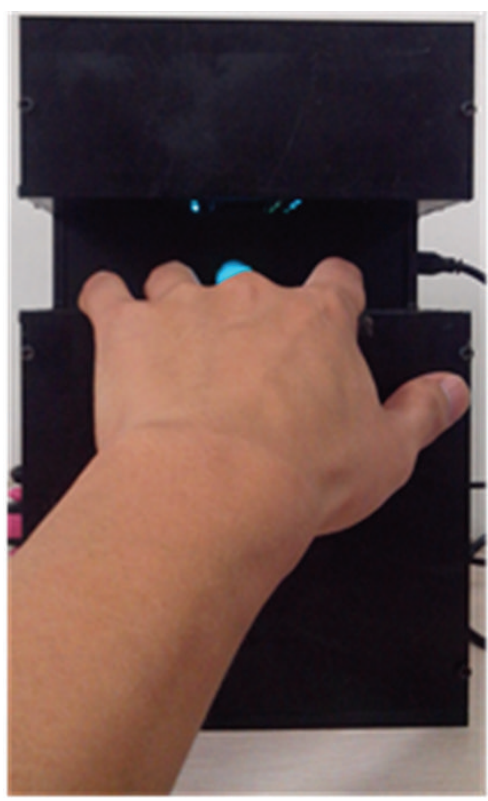

(b)

FIGURE 1: A homemade imaging system ((a) the proposed imaging principle and (b) an imaging device).

extraction. GOM integrates distinctiveness of Gabor features and robustness of ordinal measures to describe the image texture features. First, a bank of even-symmetric Gabor filters with 8 orientations [19] is used to exploit the magnitude features in FP, FV, and FKP images. Second, the ordinal filters [17] are conducted with the 8-orientation magnitude features to obtain the three-modal GOM feature maps. Third, combining the GOM feature maps into a color-feature map, we constitute the original feature object set of a finger. Forth, the original feature object set is granulated into nonoverlapping circular granules in a bottom-up manner based on spatial circular granulation to construct the multilevel feature granules (FGs). Finally, a top-down matching method is proposed to test the performance of multilevel FGs. Experimental results show that the proposed method yields high identification accuracy in finger feature recognition.

The rest of this paper is organized as follows. Section 2 introduces a homemade imaging system for the multimodal finger images acquisition. Section 3 presents a general framework of finger feature granulation based on spatial circular granulation. In Section 4, a self-built database with three modalities is used to validate the feasibility and effectiveness of the proposed algorithm; several experimental results are also reported in this section. Section 5 concludes this paper.

\section{Multimodal Finger Image Acquisition}

To obtain FP, FV, and FKP images, we have designed a homemade imaging system, which can capture these three modality images automatically and simultaneously when a finger is available, as shown in Figure 1. In the proposed imaging system, a novel double-spectral polarized imaging system is proposed. Based on the physiological structure of fingers and its imaging property, the finger-knuckle-prints and fingerprints are imaged by reflected lights, and the finger veins are imaged using the near infrared light in a transillumination manner, as shown in Figure 1(a).

Connected to the imaging device with a computer, we can readily capture FP, FV, and FKP images using a specifically designed software, as shown in Figure 2(a). After the image is captured, it is sent to the data preprocessing module for preprocessing. To extract the ROIs of the three modality images, we, respectively, apply a method proposed in [7] for FKP ROI extraction, a method proposed in [20] for FP ROI extraction, and a method in [19] for FV ROI extraction. Then we obtain a group of $96 * 208$-pixel images of FV-ROI, $96 * 208$-pixel images of the FKP-ROI, and $160 * 160$-pixel images of the FP-ROI. The results are, respectively, shown in Figure 2(b).

\section{Feature Extraction and Granulation}

3.1. Feature Extraction. Feature extraction is essential for original feature domain construction and finger feature granulation. The three-modal finger features all bear special textures that can be viewed as signatures of finger. Therefore, the used feature extraction method should be powerful in describing the image texture feature.

First, all ROI images of FP, FV, and FKP are normalized to $162 * 162$ pixels, as shown in the top row of Figure 3, respectively. Due to different texture structure of the three modalities, we use Gabor filter [19] to enhance FV and Steerable filter [21] for FP and FKP enhancement to strengthen the 


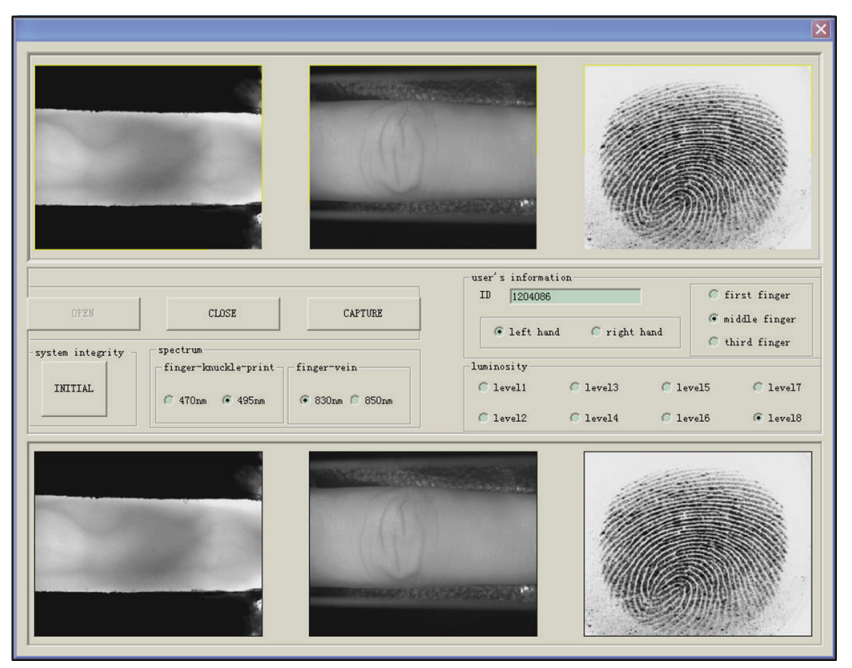

(a)
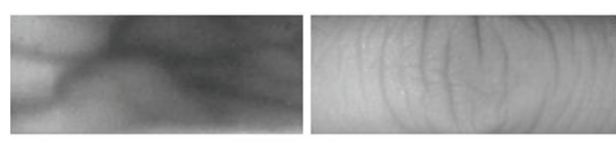

(b)

FIGURE 2: (a) A developed software interface of image acquisition. (b) The extracted ROI images (left: FV, middle: FKP, and right: FP).

blurred images, as shown in the middle row of Figure 3. Then, we use the Gabor filters with 8 orientations [19] to exploit Gabor magnitude features in the FP, FV, and FKP images:

$$
\begin{aligned}
& G(x, y) \\
& \quad=\frac{\gamma}{2 \pi \sigma^{2}} \exp \left\{-\frac{1}{2}\left(\frac{x_{\theta_{k}}^{2}+\gamma^{2} y_{\theta_{k}}^{2}}{\sigma^{2}}\right)\right\} \exp \left(\widehat{j} 2 \pi f_{k} x_{\theta_{k}}\right) .
\end{aligned}
$$

Here, $\left[\begin{array}{l}x_{\theta_{k}} \\ y_{\theta_{k}}\end{array}\right]=\left[\begin{array}{cc}\cos \theta_{k} & \sin \theta_{k} \\ -\sin \theta_{k} & \cos \theta_{k}\end{array}\right]\left[\begin{array}{l}x \\ y\end{array}\right], k$ is the number of orientations, $\sigma$ and $\gamma$, respectively, represent the scale of Gabor filter and aspect ratio of the elliptical Gaussian envelope, and $x_{\theta}$ and $y_{\theta}$ are two rotated versions of the coordinates $x$ and $y$.

Finally, the ordinal measures are conducted with the multichannel Gabor magnitude features to get the ordinal feature maps $[17,18]$, as shown in the last row of Figure 3 . The difference filter is defined as

$$
\begin{aligned}
\operatorname{MLDF}= & C_{p} \sum_{i=1}^{N_{p}} \frac{1}{\sqrt{2 \pi \delta_{p i}}} \exp \left[\frac{-\left(X-\omega_{p i}\right)^{2}}{2 \delta_{p i}^{2}}\right] \\
& -C_{n} \sum_{j=1}^{N_{n}} \frac{1}{\sqrt{2 \pi \delta_{n j}}} \exp \left[\frac{-\left(X-\omega_{n j}\right)^{2}}{2 \delta_{n j}^{2}}\right] .
\end{aligned}
$$

Here $\omega$ is the central position and $\delta$ is the scale of ordinal filter. $N_{p}$ is the number of positive lobes and $N_{n}$ is the number of negative lobes. Constant coefficients $C_{n}$ and $C_{p}$ are used to keep the balance between positive and negative lobes. To satisfy $C_{p} N_{p}=C_{n} N_{n}$, we assume $C_{p}=1, N_{p}=2$ and $C_{n}=2$, $N_{n}=1$, since the difference filter with three lobes is more stable. The feature extraction scheme of GOM is capable of representing the distinctive and robust of texture features of finger images.

3.2. Feature Granulation. Granulation is one of the key issues in $\mathrm{GrC}$ for complex problem solving. In order to effectively express the feature structure of the fingers, we firstly select the original feature object set on the basis of the feature analysis. Then, we adopt a bottom-up manner to construct the multilevel feature granules. Here, FGs are generated as the following procedure.

Step 1 (initializing). Based on feature-based registration of the three modalities, we combined FP, FV, and FKP feature maps in a color-based manner to form the RGB-GOM feature map, as shown in Figure 4. This map is used to constitute the original feature object set of a finger. Then we defined $O_{0}=(x, y, R, G, B)$, where $x, y$ are coordinate value and $R, G$, and $B$ denote the FP, FV, and FKP feature maps, respectively.

$\mathrm{O}_{1}$ represents the original feature object set, which is defined as

$$
O_{1}=\left\{\bigcup_{\mathrm{FP}}\left\{O_{i, j}\right\}\right\} \bigcap\left\{\bigcup_{\mathrm{FV}}\left\{O_{i, j}\right\}\right\} \bigcap\left\{\bigcup_{\mathrm{FKP}}\left\{O_{i, j}\right\}\right\} .
$$

Here, structure of 0-layer feature granules is constructed by a 2-tuple $G_{0}{ }^{1}=\left(I G_{0}{ }^{1}, E G_{0}{ }^{1}\right)$; the intension and extension of 0-layer feature granules are, respectively, defined as $I G_{0}{ }^{1}=$ $(x, y, \operatorname{map} R, \operatorname{map} G, \operatorname{map} B), E G_{0}{ }^{1}=\left\{x \mid x \in O_{1}\right\}$. Here, $I G_{l}{ }^{1}$ is the intension of granules in $l$-layer and $E G_{l}{ }^{1}$ is the extension of granules in $l$-layer.

Step 2 (calculating 1-layer granule $\left.G_{1}^{1}=\left(I G_{1}^{1}, E G_{1}^{1}\left(\eta_{1}^{1} \mid \operatorname{tr}_{1}^{1}\right)\right)\right)$. According to the merger rules of the tolerance granularity grid, each 1-layer granule's extension can be generated by the definition $E G_{1}^{1}\left(\eta_{1}^{1} \mid \operatorname{tr}_{1}^{1}\right)=\left\{x \mid\left(x, \eta_{1}^{1}\right) \in \operatorname{tr}_{1(\mathrm{cp}, w, \mathrm{DIS}, D)}^{1}\right\}$; here, $c p, w$, DIS, and $D$ are the four elements of the compatible function, $\mathrm{cp}$ is the compound compatibility relation proposition, $w$ is the weight coefficient of each component, DIS is distance function, and $D$ is the vector of distance function. We use the circular granule as the shape of the FGs. In this way, FGs have the translation and rotation invariant characteristics. Let $\operatorname{dis}(\alpha, \beta \omega)=\sum_{i=0}^{n-1} \omega_{i}\left(\alpha_{i}-\beta_{i}\right)^{2}$; $\operatorname{cp}(\alpha, \beta \mathrm{DIS}, D)=\operatorname{dis}(\alpha, \beta \omega) \leq d$, where $\omega=(1,1,0,0,0)$, DIS $=\{$ dis $\}, D=\{d\}, \eta_{1}^{1} \in \operatorname{Grid}_{1}{ }^{1}$, and $\operatorname{Grid}_{1}{ }^{1}$ is a grid point set, which includes all centers of granules $G_{1}^{1}, d=a^{2}$ $(a=4)$. The histogram of the ordinal code as the intension of FGs $I G_{1}^{1}=\left(H_{1}, H_{2}, \ldots, H_{L}\right)$, and $L$ is the bin number of the histograms. Thus, the original feature object set is granulated as circular granules with the radius of 4 pixels, denoted as 1layer FGs:

$$
\text { radius }=\text { floor }\left(\frac{\text { pixels of the row }}{\text { the number of circular granules }}\right) .
$$



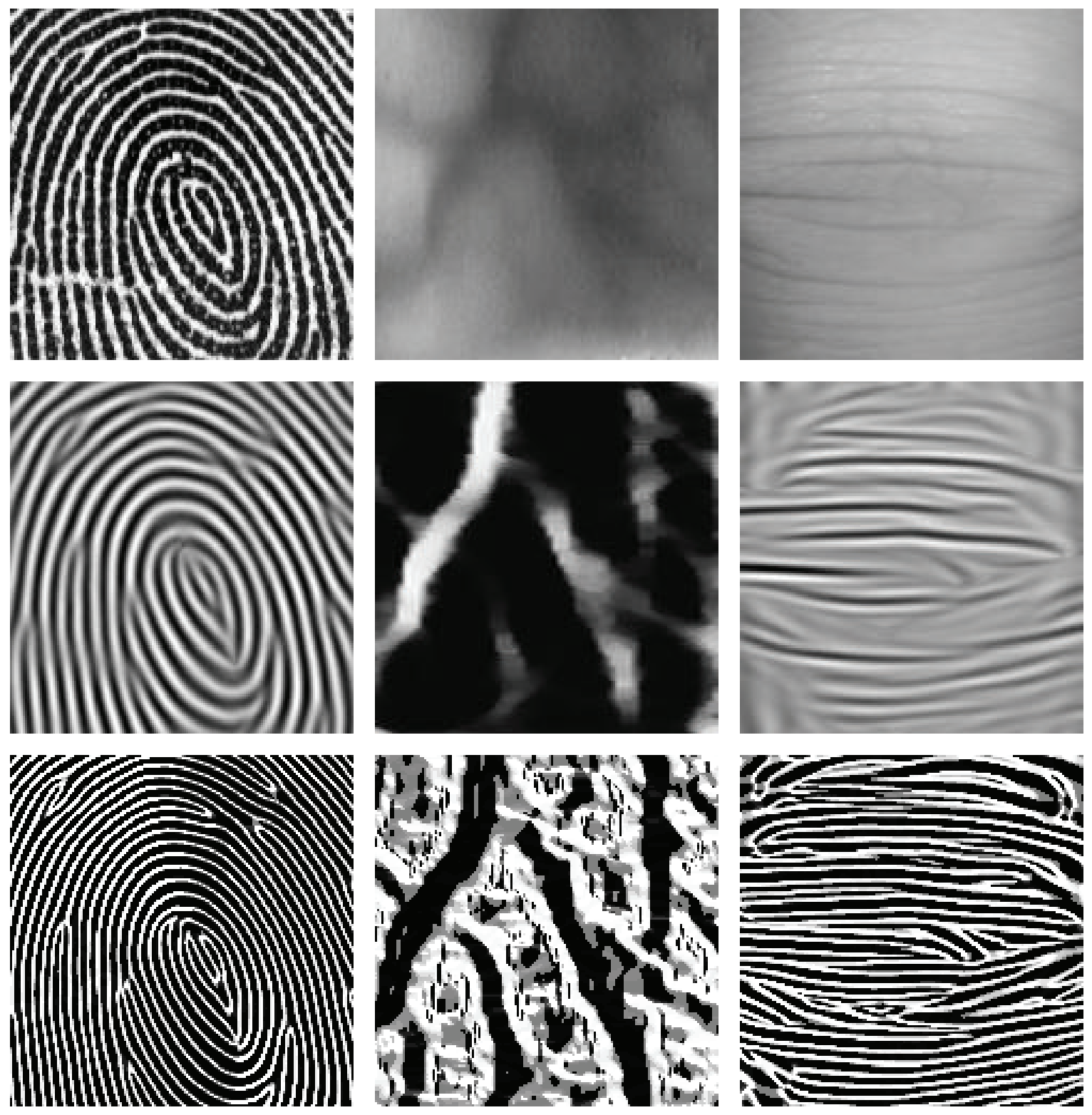

FIGURE 3: Gabor Ordinal Measures feature maps of the FP, FV, and FKP.

Step 3 (calculating $\left.G_{i+1}^{1}=\left(I G_{i+1}^{1}, E G_{i+1}^{1}\left(\eta_{i+1}^{1} \mid \operatorname{tr}_{i+1}^{1}\right)\right)\right)$. Recursively, calculating the $i+1$-layer feature granules, $G_{i+1}^{1}=$ $\left(I G_{i+1}^{1}, E G_{i+1}^{1}\left(\eta_{i+1}^{1} \mid \operatorname{tr}_{i+1}^{1}\right)\right)$. Extension of feature granules on the layer $i+1$ can be obtained by the following formula $E G_{i+1}^{1}\left(\eta_{i+1}^{1} \mid \operatorname{tr}_{i+1}^{1}\right)=\left\{x \mid\left(x, \eta_{i+1}^{1}\right) \in \operatorname{tr}_{i+1\left(\mathrm{cp}_{i+1}, w_{i+1}, \mathrm{DIS}_{i+1}, D_{i+1}\right)}^{1} \wedge\right.$ $\left.x \in E G_{i}^{1}\right\}$. If this step continues, the extension of the highestlevel granules eventually contains only one original object. In this paper, FGs are generated considering three granularity levels, and the three-layer bottom-up granulation process is shown in Figure 5.

\section{Experiments and Analysis}

4.1. Recognition Analysis. As we know, statistical distribution of texture primitives has become a standard description of texture analysis. Since the ordinal codes are binary values and 8-orientation Gabor filters are used, we can obtain a binary number of 8 bits. This means that there is 256-bin histogram representation for each FG. To reduce the feature dimension, the histogram of each FG can be further reduced to contain only $B=32$ bins by partitioning the histograms into uniform parts: $[0, \ldots, 256 / B-1],[256 / B, \ldots, 2 * 256 / B-1], \ldots,[(B-$ $1) * 256 / B, \ldots, B * 256 / B-1]$ [18]. To test the performance of the proposed method, the histograms computed for the FGs are concatenated here to represent different levels FGs. Here, we use the normalized histogram intersection $H\left(\mathrm{FG}^{1}, \mathrm{FG}^{2}\right)$ [22] as the similarity measurement of two FGs, then we defined the similarity between two finger images in the $i$ th layer as

$$
\operatorname{Sim}_{i}\left(F^{1}, F^{2}\right)=\sum_{r=0}^{N_{i}-1} H\left(\mathrm{FG}_{r}^{1}, \mathrm{FG}_{r}^{2}\right)
$$




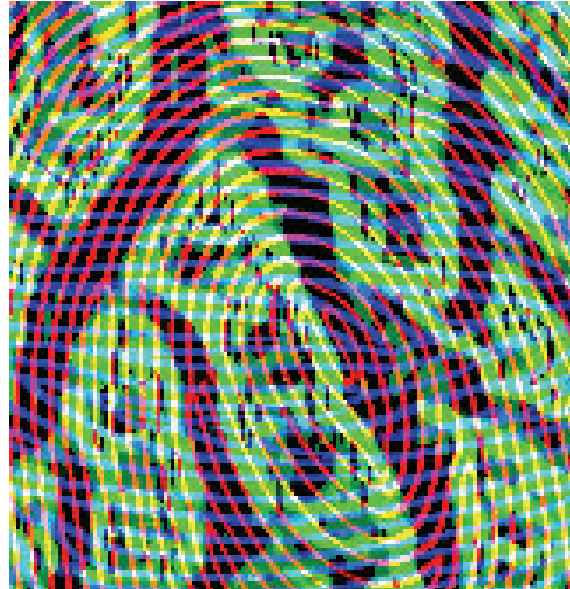

FIgURE 4: The original feature object set of a finger.

Here, $N_{i}$ is the number of granules in the $i$ th granularity level. If $\operatorname{Sim}_{i} \geq T_{i}\left(T_{i}\right.$ is the decision threshold of the $i$ th layer), then the two finger images match in the $i$ th layer granules. As the multimodal finger-based recognition can be addressed at multigranularity levels and the coarse granularity information is an abstract description of the finger feature, it is able to narrow the scope of problem solving and accelerate the calculating speed at the coarse granularity levels. In contrast, the fine granularity information represents a specific description of the finger feature. Hence, for the problems that cannot be solved at coarse granularity levels, we could address them in a fine granularity level. Thus, a top-down (coarse-to-fine) recognition method is proposed. Only when the two finger images matched in three granularity layers at the same time $\left(\operatorname{Sim}_{3} \geq T_{3}, \operatorname{Sim}_{2} \geq T_{2}\right.$, and $\left.\operatorname{Sim}_{1} \geq T_{1}\right)$, the two finger images are similar.

4.2. Recognition Results. In this section, we carry out several experiments to prove the feasibility and effectiveness of the proposed algorithm. A self-constructed database which totally contains $3000 \mathrm{FP}$ images, $3000 \mathrm{FV}$ images, and 3000 FKP images from 300 individuals is used in these experiments. Firstly, the comparison between the three unimodal biometrics is shown in Figure 6. From these presented curves, we can see that FV achieves a lower EER than FP and FKP, which shows that FV has more robust and reliable texture feature information.

Also, Figure 7 shows the comparison between the multimodal biometric systems. From this we can see that fusion between features which performed better in single modal will get better results. As expected, FGs yields significantly better performance compared with any single modal or bimodal fusion of FP, FV, and FKP. The above comparison shows that the proposed method can effectively achieve information complementary between different finger features. This is beneficial for identification performance improvement.

Figure 8 shows the similarity distributions of genuine and imposter matching generated by the three granularity levels. From the curves we can see that the proposed algorithm is capable of differentiating finger images by setting appropriate

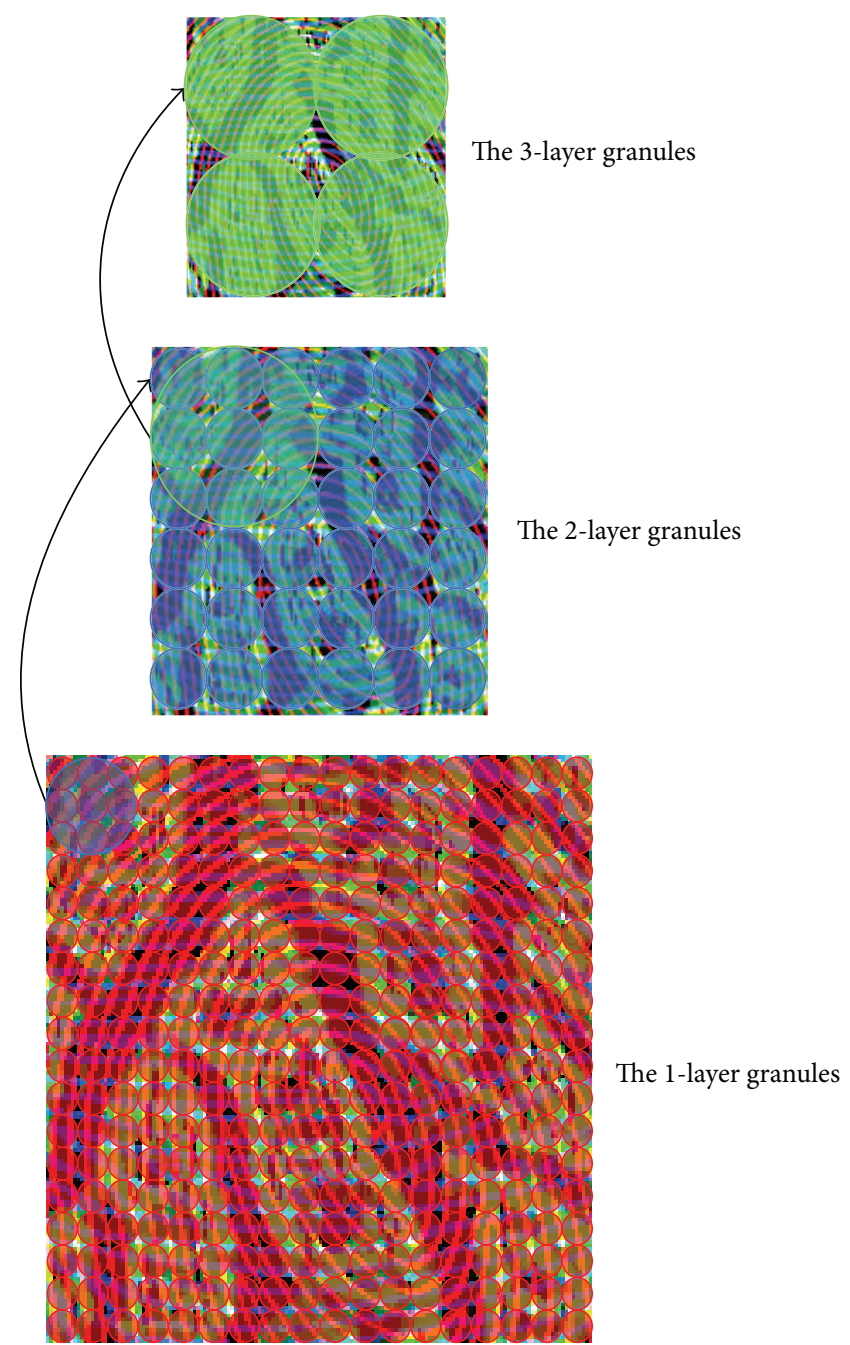

FIGURE 5: The 3-layer bottom-up granulation process.

value of the threshold. This implies that the proposed algorithm can provide a good solution for handling both intraclass variations and interclass similarity of finger images. Moreover, from these curves we can also conclude that the discrimination of 1-layer FGs (fine-grained information) is better than the two other layers (coarse-grained information).

Figure 9 shows the ROC curves generated by the different granularity levels. Here the proposed algorithm is implemented using MATLAB R2010a on a standard desktop PC which is equipped with a Dual-Core CPU $2.5 \mathrm{GHz}$ and 4 GB RAM. From these curves we can clearly see that the 1-layer FGs (1-FGs) make a lower EER compared with two other layers, indicating that the matching performance in fine-granularity space outperforms coarse granularity space. Further, Table 1 listed the matching results of three different granularity levels. From Table 1, we can draw the conclusion that the coarse-grained information has the higher matching efficiency and the fine-grained information has the higher matching accuracy. Therefore, the combination of coarse granularity information and fine-granularity information can keep the recognition efficiency in the premise of accuracy. 


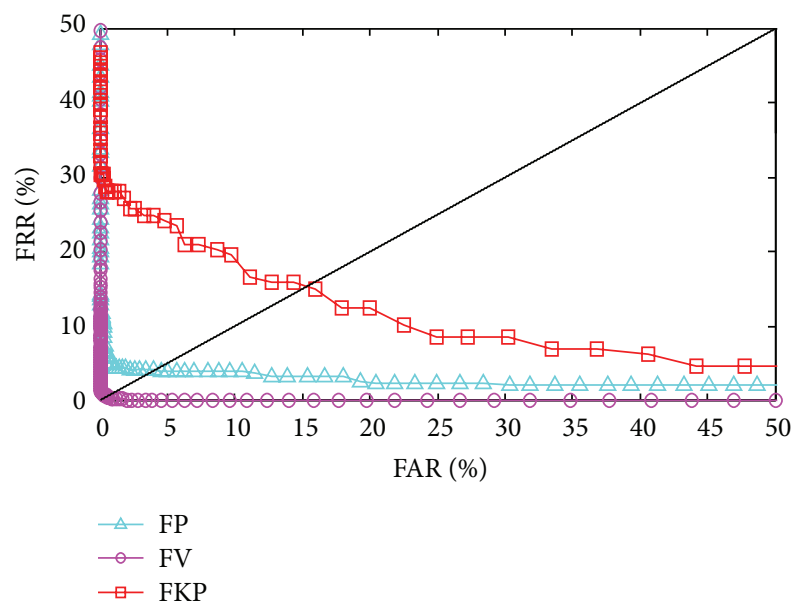

FIGURE 6: Comparisons between unimodal biometric systems.

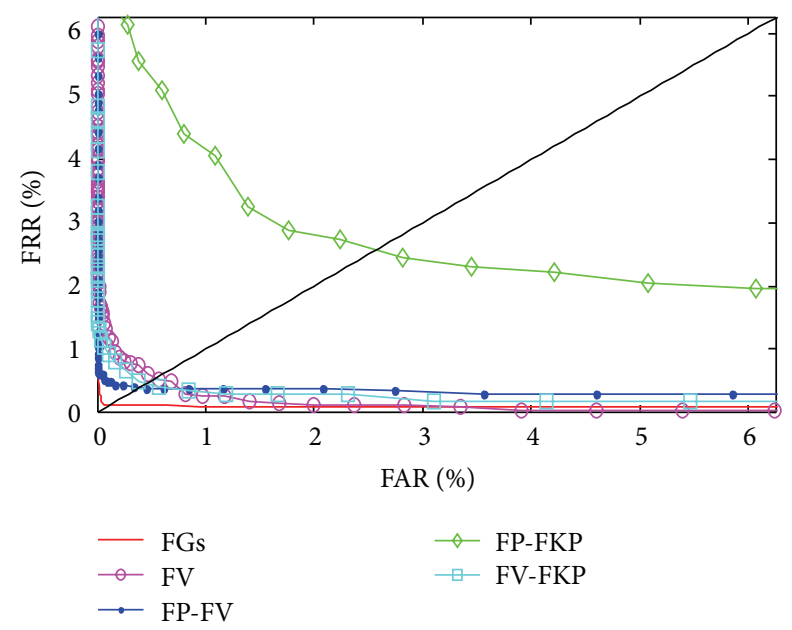

FIGURE 7: Comparisons between multimodal biometric systems.

TABLE 1: Matching results of different levels FGs.

\begin{tabular}{|c|c|c|c|}
\hline \multirow[b]{2}{*}{ Granular level } & \multicolumn{3}{|c|}{ Matching performance } \\
\hline & $\operatorname{EER}(\%)$ & $\begin{array}{c}\text { Matching } \\
\text { time (s) }\end{array}$ & $\begin{array}{c}\text { Recognition rate } \\
(\%)\end{array}$ \\
\hline One-layer & 0.853 & 0.257 & 99.15 \\
\hline Two-layer & 2.230 & 0.074 & 97.77 \\
\hline Three-layer & 3.408 & 0.028 & 96.59 \\
\hline
\end{tabular}

Further, Table 2 listed the matching performance for the top-down matching method. From the data we can see that the coarse-to-fine matching method of 3-2-1-layer FGs achieves the best accuracy recognition results. According to $T$ of 1-layer and 2-layer FGs when FAR is minimum and FRR $=0$, the threshold of 3-layer and 2-layer FGs is, respectively, set as $T_{3}=0.738$ and $T_{2}=0.689$. The multilayer top-down matching method also reduces the matching time costs since the nonmatched granules are ignored in the high granularity level. Therefore, the top-down recognition method of multilayer FGs performs better in finger-based recognition in both efficiency and accuracy. And we should
TABLE 2: Matching results of the top-down matching method.

\begin{tabular}{|c|c|c|c|}
\hline \multirow[b]{2}{*}{ Granular level } & \multicolumn{3}{|c|}{ Matching performance } \\
\hline & $\operatorname{EER}(\%)$ & $\begin{array}{c}\text { Matching } \\
\text { time (s) }\end{array}$ & $\begin{array}{c}\text { Recognition rate } \\
(\%)\end{array}$ \\
\hline 3-2-layer & 1.522 & 0.056 & 98.48 \\
\hline 2-1-layer & 0.627 & 0.096 & 99.37 \\
\hline 3-2-1-layer & 0.415 & 0.129 & 99.58 \\
\hline
\end{tabular}

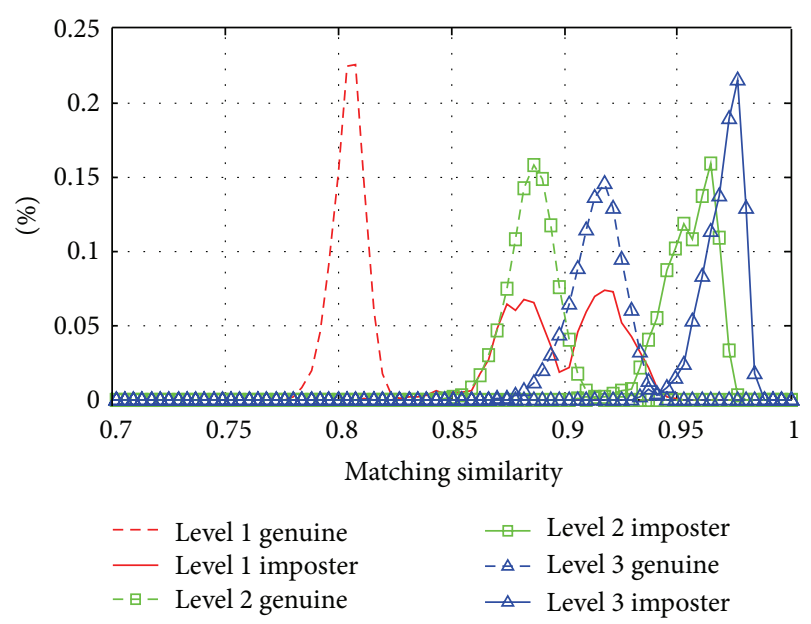

FIGURE 8: Similarity distributions of genuine and imposter matching at three granularity levels.

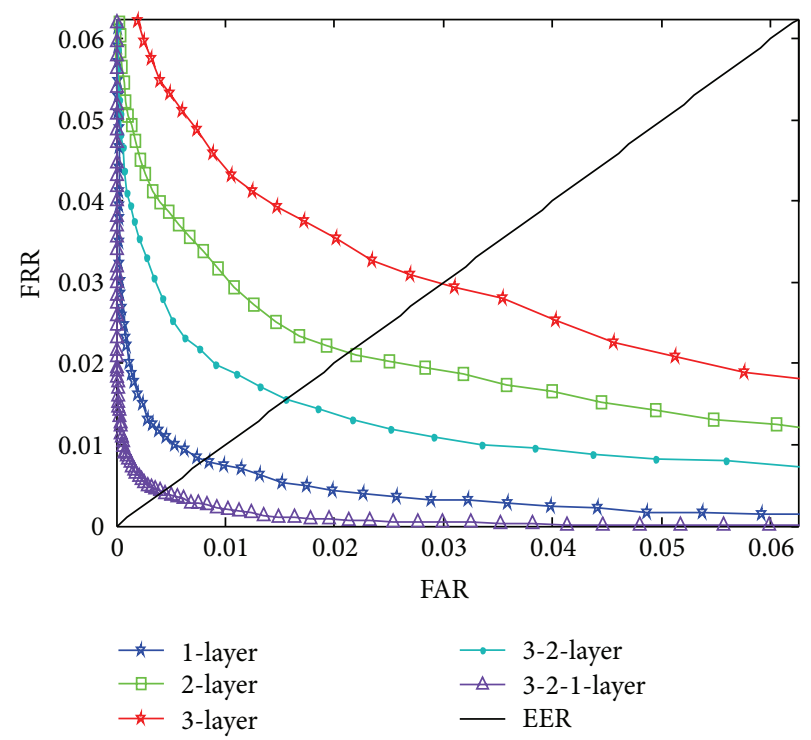

FIGURE 9: ROC curves of different granularity levels.

note that the matching time is related to the PC performance: when the run time is longer, the speed will slow down.

\section{Conclusion}

A new multimodal finger feature recognition scheme based on spatial circular granulation has been proposed in this paper. Firstly, the ridge texture features of the FP, FV, and FKP 
images were both extracted by the Gabor Ordinal Measures. Then, combining the GOM feature maps into a color-feature map, we then constitute the original feature object set of a finger. Finally, three-level FGs were constructed based on spatial circular granulation. Experimental results show that circular FGs are much more reliable and precise in multimodal finger feature recognition, and the top-down recognition method of multilevel FGs is helpful for identification performance and efficiency improvement.

\section{Competing Interests}

The authors declare that they have no competing interests.

\section{Acknowledgments}

This work is jointly supported by National Natural Science Foundation of China (no. 61379102) and the Fundamental Research Funds for the Central Universities (no. 3122014C003).

\section{References}

[1] A. Jain, L. Hong, and R. Bolle, "Online fingerprint verification," IEEE Transactions on Pattern Analysis and Machine Intelligence, vol. 19, no. 4, pp. 302-314, 1997.

[2] A. K. Jain, Y. Chen, and M. Demirkus, "Pores and ridges: highresolution fingerprint matching using level 3 features," IEEE Transactions on Pattern Analysis and Machine Intelligence, vol. 29, no. 1, pp. 15-27, 2007.

[3] E. C. Lee, H. C. Lee, and K. R. Park, "Finger vein recognition using minutia-based alignment and local binary pattern-based feature extraction," International Journal of Imaging Systems and Technology, vol. 9, pp. 179-186, 2009.

[4] J. Yang, Y. Shi, and J. Yang, "Finger-vein recognition based on a bank of gabor filters," in Proceedings of the Asian Conference on Computer Vision, pp. 374-383, Xian, China, September 2009.

[5] J. Yang, Y. Shi, and J. Yang, "Personal identification based on finger-vein features," Computers in Human Behavior, vol. 27, no. 5, pp. 1565-1570, 2011.

[6] L. Zhang, L. Zhang, D. Zhang, and H. Zhu, "Ensemble of local and global information for fingerknuckle-print recognition," Pattern Recognition, vol. 44, no. 9, pp. 1990-1998, 2011.

[7] L. Zhang, L. Zhang, D. Zhang, and H. Zhu, "Online fingerknuckle-print verification for personal authentication," Pattern Recognition, vol. 43, no. 7, pp. 2560-2571, 2010.

[8] A. Ross and A. Jain, "Information fusion in biometrics," Pattern Recognition Letters, vol. 24, no. 13, pp. 2115-2125, 2003.

[9] A. Ross and A. K. Jain, "Multimodal biometrics: an overview," in Proceedings of the 12th European Signal Processing Conference, pp. 1221-1224, Vienna, Austria, September 2004.

[10] J. Yang and X. Zhang, "Feature-level fusion of fingerprint and finger-vein for personal identification," Pattern Recognition Letters, vol. 33, no. 5, pp. 623-628, 2012.

[11] D. Miao, G. Wang, and Q. Liu, Granular Computing: Past, Present and Prospect, Science Publishing House, Beijing, China, 2007 (Chinese).

[12] J. T. Yao, A. V. Vasilakos, and W. Pedrycz, "Granular computing: perspectives and challenges," IEEE Transactions on Cybernetics, vol. 43, no. 6, pp. 1977-1989, 2013.
[13] Z. Zheng, H. Hu, and Z. Z. Shi, "Tolerance granular space and its applications," in Proceedings of the IEEE International Conference on Granular Computing, pp. 367-372, Beijing, China, July 2005.

[14] Z. Shi, Z. Zheng, and Z. Meng, "Image segmentation-oriented tolerance granular computing model," in Proceedings of the IEEE International Conference on Granular Computing, pp. 566-571, IEEE, Hangzhou, China, August 2008.

[15] Z. Li and Z. Meng, "Technique of medical image fusion based on tolerance granular space," Application Research of Computers, vol. 27, pp. 1192-1194, 2010.

[16] H. S. Bhatt, S. Bharadwaj, R. Singh, and M. Vatsa, "Recognizing surgically altered face images using multiobjective evolutionary algorithm," IEEE Transactions on Information Forensics and Security, vol. 8, no. 1, pp. 89-100, 2013.

[17] Z. Sun, T. Tan, Y. Wang, and S. Z. Li, "Ordinal palmprint represention for personal identification," in Proceedings of the IEEE Computer Society Conference on Computer Vision and Pattern Recognition (CVPR '05), vol. 1, pp. 279-284, San Diego, Calif, USA, June 2005.

[18] Z. Chai, Z. Sun, H. Mendez-Vazquez, R. He, and T. Tan, "Gabor ordinal measures for face recognition," IEEE Transactions on Information Forensics and Security, vol. 9, no. 1, pp. 14-26, 2014.

[19] J. Yang and Y. Shi, "Finger-vein ROI localization and vein ridge enhancement," Pattern Recognition Letters, vol. 33, no. 12, pp. 1569-1579, 2012.

[20] H. B. Kekre and V. A. Bharadi, "Fingerprint's core point detection using orientation field," in Proceedings of the International Conference on Advances in Computing, Control and Telecommunication Technologies (ACT '09), pp. 150-152, IEEE, Kerala, India, December 2009.

[21] W. T. Freeman and E. H. Adelson, "The design and use of steerable filters," IEEE Transactions on Pattern Analysis and Machine Intelligence, vol. 13, no. 9, pp. 891-906, 1991.

[22] M. J. Swain and D. H. Ballard, "Color indexing," International Journal of Computer Vision, vol. 7, no. 1, pp. 11-32, 1991. 


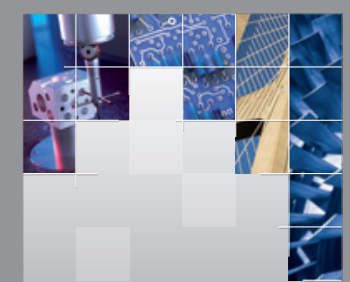

\section{Enfincering}
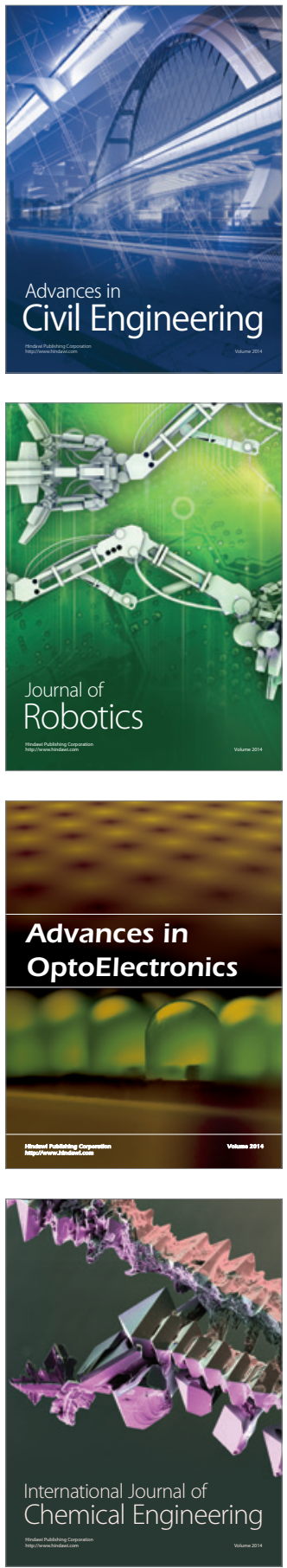

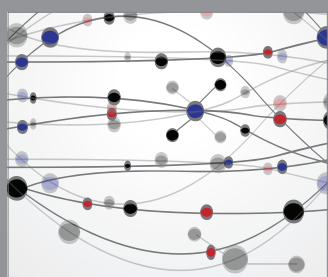

The Scientific World Journal

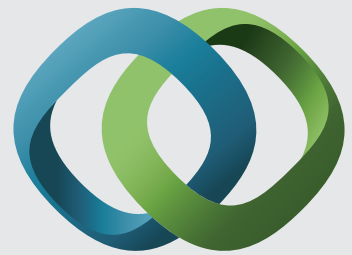

\section{Hindawi}

Submit your manuscripts at

http://www.hindawi.com
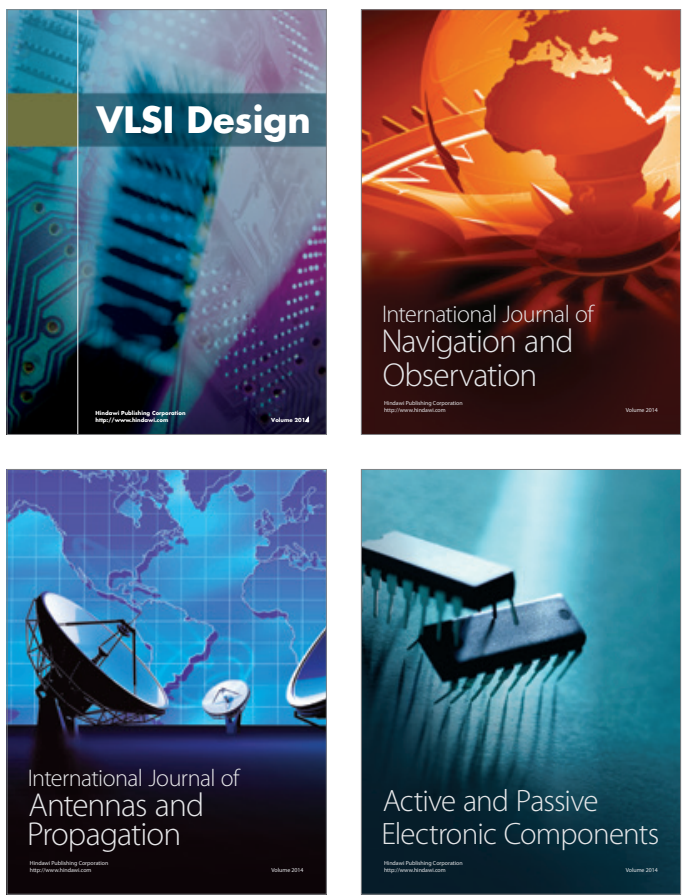
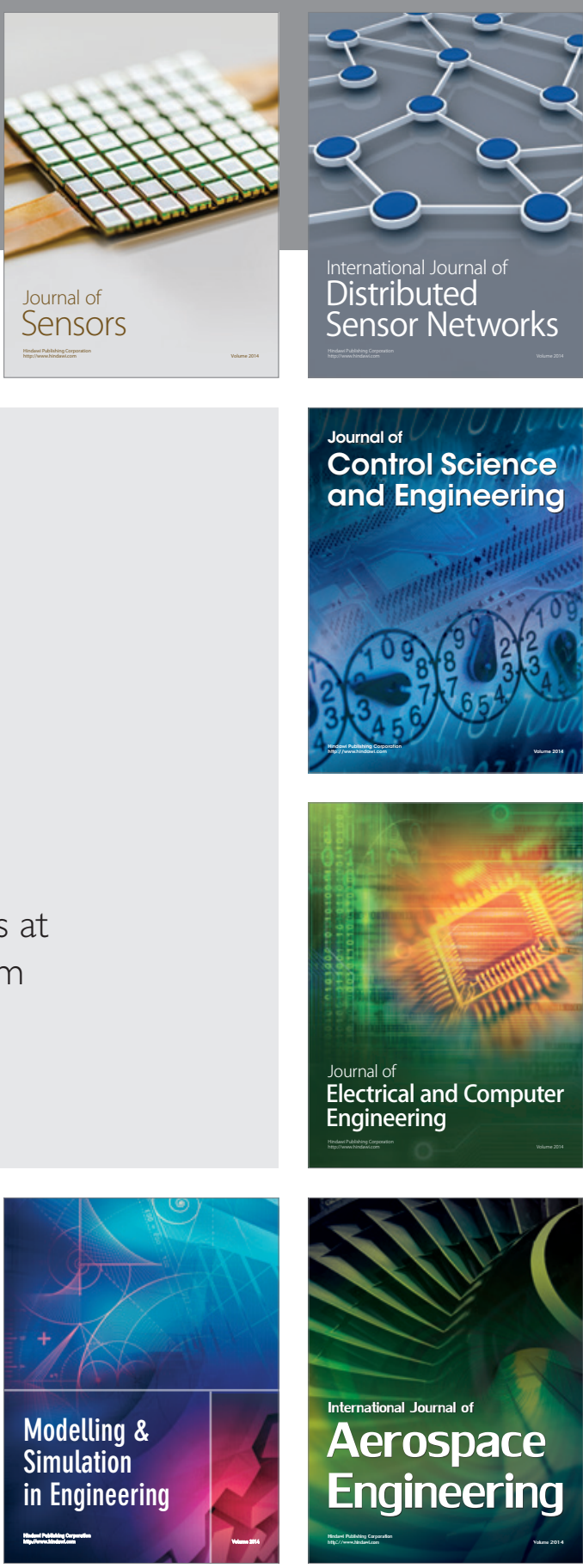

International Journal of

Distributed

Sensor Networks

Journal of

Control Science

and Engineering
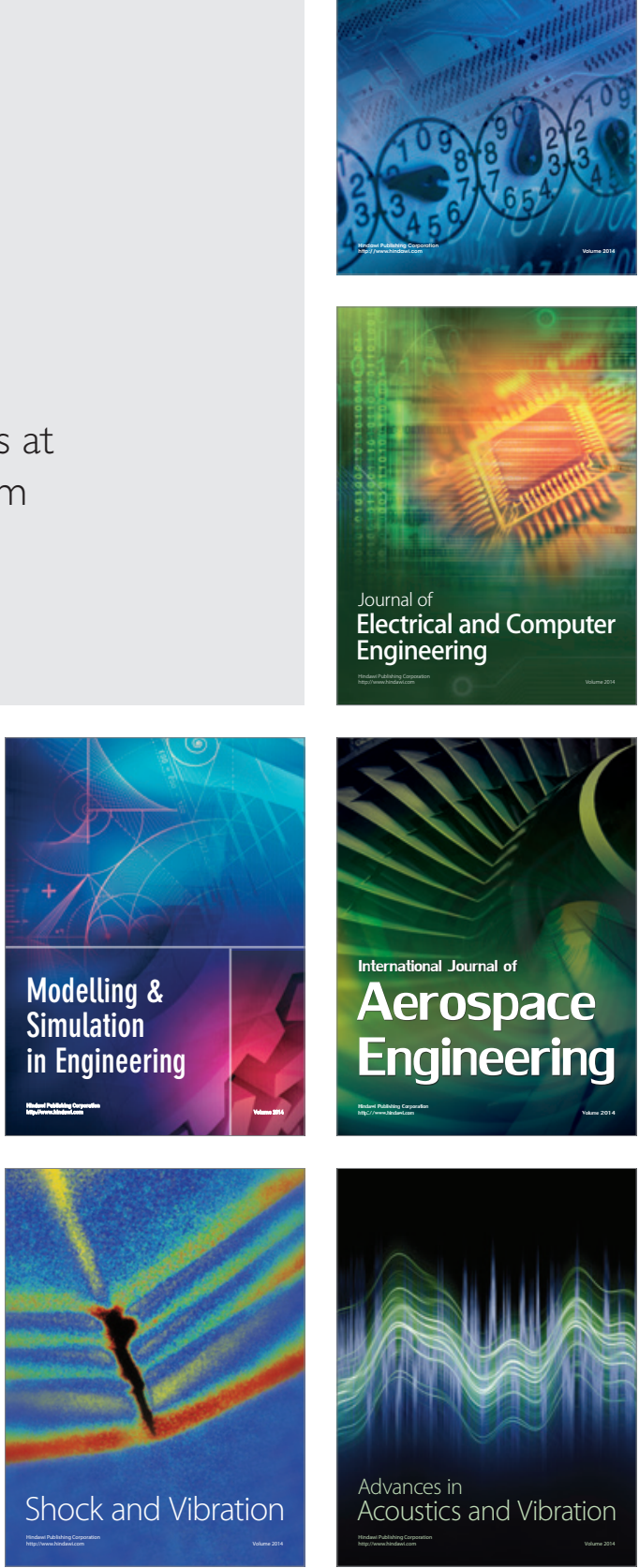